Revue des patrimoines

\title{
Le patrimoine « in silico ». Exemple de la cathédrale d'Amiens
}

The 'in silico' heritage, the example of the cathedral of Amiens

\section{El Mustapha Mouaddib, Guillaume Caron, Dominique Groux-Leclet et Fabio Morbidi}

\section{(2) OpenEdition}

Journals

Édition électronique

URL : http://journals.openedition.org/insitu/21576

DOI : 10.4000/insitu.21576

ISSN : 1630-7305

Éditeur

Ministère de la Culture

Référence électronique

El Mustapha Mouaddib, Guillaume Caron, Dominique Groux-Leclet et Fabio Morbidi, « Le patrimoine « in silico ». Exemple de la cathédrale d'Amiens », In Situ [En ligne], 39 | 2019, mis en ligne le 24 mai 2019, consulté le 11 juillet 2019. URL : http://journals.openedition.org/insitu/21576 ; DOI : 10.4000/ insitu. 21576

Ce document a été généré automatiquement le 11 juillet 2019.

\section{(i) $\odot$}

In Situ Revues des patrimoines est mis à disposition selon les termes de la licence Creative Commons Attribution - Pas d'Utilisation Commerciale - Pas de Modification 4.0 International. 


\title{
Le patrimoine « in silico ». Exemple de la cathédrale d'Amiens
}

\author{
The 'in silico' heritage, the example of the cathedral of Amiens \\ El Mustapha Mouaddib, Guillaume Caron, Dominique Groux-Leclet et \\ Fabio Morbidi
}

Le programme E-Cathédr@le est soutenu financièrement par la région Hauts-de-France et les fonds FEDER. Les partenaires du programme sont : Amiens Métropole, la DRAC des Hauts-de-France et le Centre des monuments nationaux. La numérisation a fait l'objet d'une collaboration avec l'IGN (Institut national de l'information géographique et forestière) et l'ENSG (École Nationale des Sciences Géomatiques) d'octobre 2010 à octobre 2012.

\section{Introduction ${ }^{1}$}

1 Le développement des techniques de numérisation d'une part et celui des outils informatiques d'autre part ouvrent des perspectives extraordinaires dans tous les domaines. Le patrimoine monumental en fait partie. Il en constitue même un terrain privilégié par la nature de ses enjeux sociétaux, économiques et scientifiques. Mais si l'intérêt perçu immédiatement fait consensus et est estimé à sa juste valeur, la mise en œuvre de tout projet mobilisant le numérique au service du patrimoine reste un exercice coûteux, difficile, chronophage, périlleux et délicat. Malgré ces difficultés, plusieurs initiatives émanant d'organismes publics, de structures académiques et d'initiatives personnelles ont vu le jour. Parmi celles-ci, l'opération portée par l'ENSAM sur l'abbaye de Cluny constitue un projet précurseur (premiers travaux en 1992) de valorisation du patrimoine par la 3D, qui a mis au point des dispositifs novateurs et pertinents comme la visualisation 2D à l'aide d'écrans situés à l'extérieur et révélant l'abbatiale de Cluny aux visiteurs.

2 D'un point de vue plus institutionnel, on peut citer l'initiative du ministère de la Culture et de la Communication qui a mis en place le programme national de numérisation 3D du patrimoine (3D-Monuments, 2003-2010) dont la maitrise d'œuvre a été confiée au 
laboratoire $\mathrm{MAP}^{2}$. Ce programme avait un double objectif: la conservation et la valorisation par le numérique. Il a permis de numériser plusieurs sites et œuvres. En outre, des appels à projets publiés régulièrement ont donné lieu à la numérisation de fonds documentaires 2D et d'œuvres en 3D sur l'ensemble du territoire: l'expérience accumulée aboutit désormais à des préconisations et des cahiers des charges qui ont vocation à aider les commanditaires de la numérisation. À titre d'exemple, le lecteur peut se référer aux documents édités par le ministère de la Culture et de la Communication ${ }^{3}$ et au livre blanc ${ }^{4}$ publié par le consortium 3D SHS. Ces recommandations accompagnent le recours intensif à la 3D dans tous les secteurs applicatifs et dans la recherche, qu'elle relève des sciences humaines et sociales ou pas.

3 Malgré cet usage désormais très répandu d'outils de la 3D et les nombreux projets de recherche nationaux, européens et internationaux, il y a encore très peu de projets voués à la numérisation complète de monuments architecturaux comme les cathédrales. Quand ils existent, ils sont rarement inscrits dans une temporalité compatible avec les exigences de cette problématique. Parmi les raisons de cette absence, il y a d'abord les modes de financement et la culture du projet qui régit désormais toute initiative, obligeant les acteurs à borner leurs travaux dans des durées n'excédant que très rarement quatre années. Ensuite, il y a le manque de pérennisation des financements dédiés à ces projets. Enfin, la difficulté à valoriser les travaux de numérisation sur le plan académique est un facteur à ne pas négliger.

4 Il est pourtant nécessaire de rompre avec ce paradigme d'une recherche pilotée par le projet et par la course à la nouveauté pour réussir les numérisations d'envergure. Cette nécessité était pour nous une évidence qu'il a fallu envisager dès le départ pour la numérisation de la cathédrale d'Amiens et qui a donné lieu au programme E-Cathédr@le.

\section{Le programme E-Cathédr@le}

$5 \quad$ E-Cathédr@le $e^{5}$ est un programme de recherche et de développement qui a démarré en 2010 et qui devrait se dérouler sur quinze ans. En constatant le manque de documents techniques précis et sûrs, comme des dessins et des plans d'élévation, ses acteurs ont émis l'idée de réaliser un relevé 3D complet de l'ensemble de la cathédrale d'Amiens, intérieur et extérieur, le plus précis, le plus résolu et le plus fidèle possible au monument tel qu'il est actuellement. À partir de ce relevé (sous forme d'un modèle 3D), plusieurs déclinaisons peuvent être envisagées par simplification du modèle en fonction des usages. Ainsi, l'objet réalisé doit pouvoir servir à des spécialistes comme les historiens de l'art, les archéologues, les architectes et les historiens. Il doit aussi servir à effectuer une sauvegarde numérique du monument, à le rendre plus accessible par des visites virtuelles et enfin, pouvoir être utilisé comme «terrain» expérimental pour des travaux de recherche de numérisation de masse ou des travaux de recherche pluridisciplinaire. Audelà de la numérisation de la cathédrale d'Amiens, le programme ambitionnait donc dès ses débuts de créer des synergies entre les différents acteurs du patrimoine. Ces objectifs, a priori inconciliables, ont constitué l'âme du programme E-Cathédr@le et justifient sa durée de réalisation, prévue dès le démarrage du programme.

6 D’un point de vue opérationnel, E-Cathédr@le est structuré autour de quatre axes. L'axe Numérisation englobe la production de données, les travaux sur les capteurs et sur les vecteurs de ces capteurs. L'axe Modèles s'intéresse à la question de la représentation des données, des attributs, de l'apparence et de la multimodalité. Dans l'axe Structures, nous 
travaillons, en collaboration avec des historiens de l'art, sur l'exploitation du modèle 3D pour la compréhension du bâti, de son histoire ${ }^{6}$ et en particulier, de l'histoire de sa construction. Enfin, l'axe Usages interroge la question des usages et la place des usagers dans les dispositifs de médiation et de valorisation des modèles 3D que nous produisons. Puisque nous allons surtout développer ici les aspects liés à la numérisation, nous renvoyons les lecteurs à nos précédents articles ${ }^{7}$ à propos des travaux que nous avons menés dans cet axe, sur les jeux sérieux et sur un système d'aide à la navigation pertinente dans les nuages de points très denses guidée par le contenu des images.

7 Des projets de développement d'applications pour la médiation, des projets de recherche et des campagnes de numérisation et de production de données viennent alimenter ces axes.

8 Les approches techniques et scientifiques de la numérisation sont désormais des choses bien établies et connues et nous nous contenterons de citer celles que nous avons utilisées. Il s'agit de la lasergrammétrie, de la photogrammétrie et de la modélisation par un modeleur (logiciel de modélisation 3D par dessin). Le lecteur peut se reporter à l'article de E. Baltsavias ${ }^{8}$ pour un retour d'expérience et des comparaisons entre ces techniques. En ce qui concerne E-Cathédr@le, la lasergrammétrie est de loin la méthode qui a été la plus utilisée. La lasergrammétrie permet de réaliser la numérisation même si les conditions d'illumination et d'accès sont très difficiles. Elle a aussi permis de numériser les hauteurs sans difficulté particulière et sans avoir recours à des vecteurs aériens (drone, avion ...). Deux types de scanner ont été utilisés : le C10 (Leica) et le Focus 3D de Faro. De manière générale, nous utilisons le $\mathrm{C} 10$ pour les grands volumes et le Focus 3D pour les espaces exigus comme les combles ou bien encore les chapelles. Ainsi, entre octobre 2010 et mars 2018 (date de rédaction de cet article), environ 1000 scans ont été réalisés à partir du sol intérieur (nef, collatéraux, chapelles, chœur), du sol extérieur, du triforium, de l'extérieur au niveau des toitures des chapelles, de l'extérieur au niveau de la toiture, de l'intérieur des charpentes et des combles. Ces scans, qui représentent près de 20 milliards de points, ont permis de couvrir environ $80 \%$ de la surface totale (sols, murs, vitraux, charpentes, toiture, combles...). La résolution obtenue varie entre $2 \mathrm{~mm}$ et $5 \mathrm{~mm}$. La texture actuelle des points 3D est obtenue en utilisant les couleurs délivrées par les caméras intégrées au scanner.

9 Nous présentons dans la figure 1 un rendu de la façade obtenu à partir du modèle actuel et une photographie de la façade pour comparaison (fig. 1). 
Figure 1
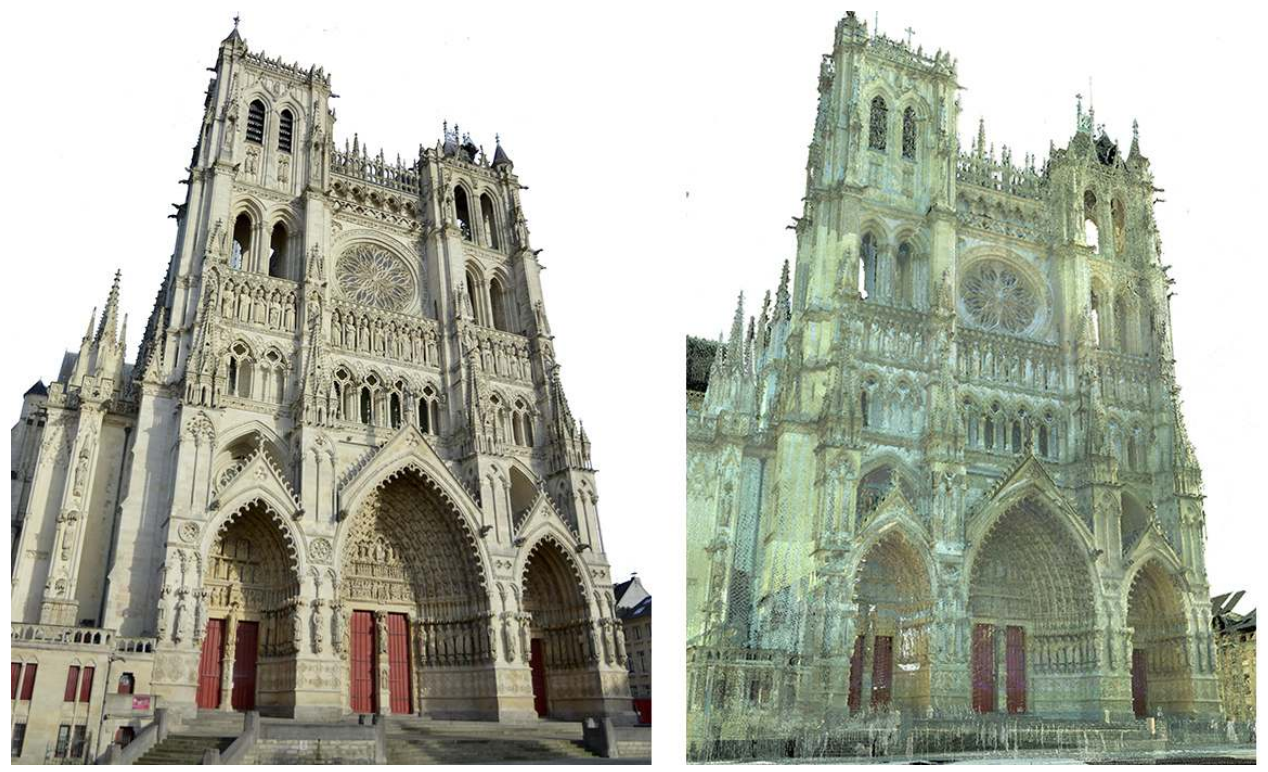

À gauche une photographie de la façade occidentale de la cathédrale d'Amiens. À droite, un rendu du modèle 3D à partir d'un point de vue similaire à celui de la photo.

(c) Laboratoire MIS (programme E-Cathédr@le).

10 La figure 2 présente un rendu de la cathédrale vue à partir du jardin de l'évêché (NordEst) et un rendu de l'enveloppe interne de la cathédrale (les points relevés de l'intérieur de la cathédrale) (fig. 2).

Figure 2

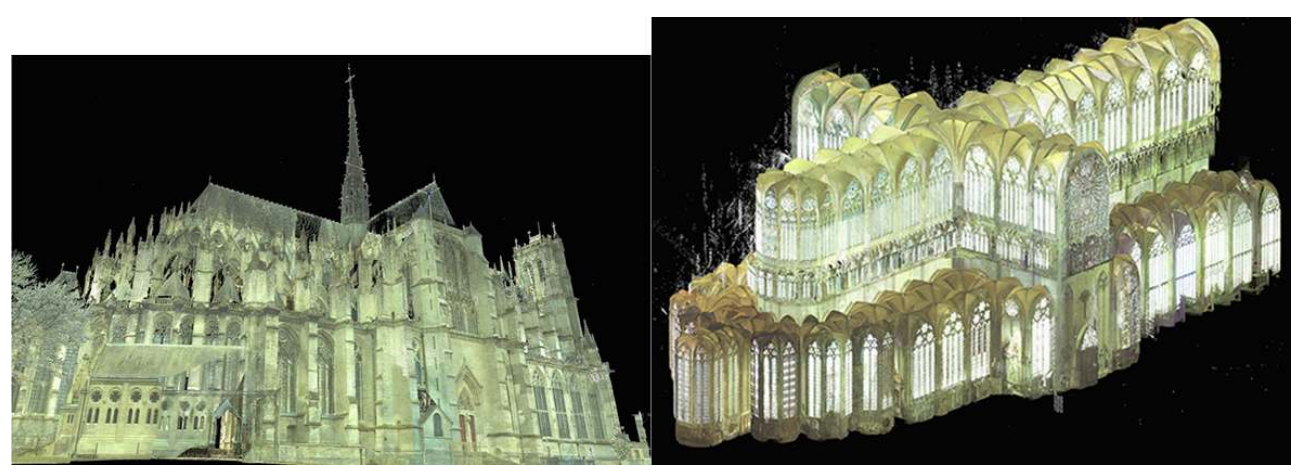

À gauche, un rendu à partir du nord-est. À droite, un rendu de l'enveloppe interne de la cathédrale (tout ce qui a été scanné à partir de l'intérieur).

(c) Laboratoire MIS (programme E-Cathédr@le).

11 La complétude et l'exigence imposée depuis le démarrage du programme permettent de générer des plans et des élévations comme on peut le voir sur la figure 3 (fig. 3). 
Figure 3

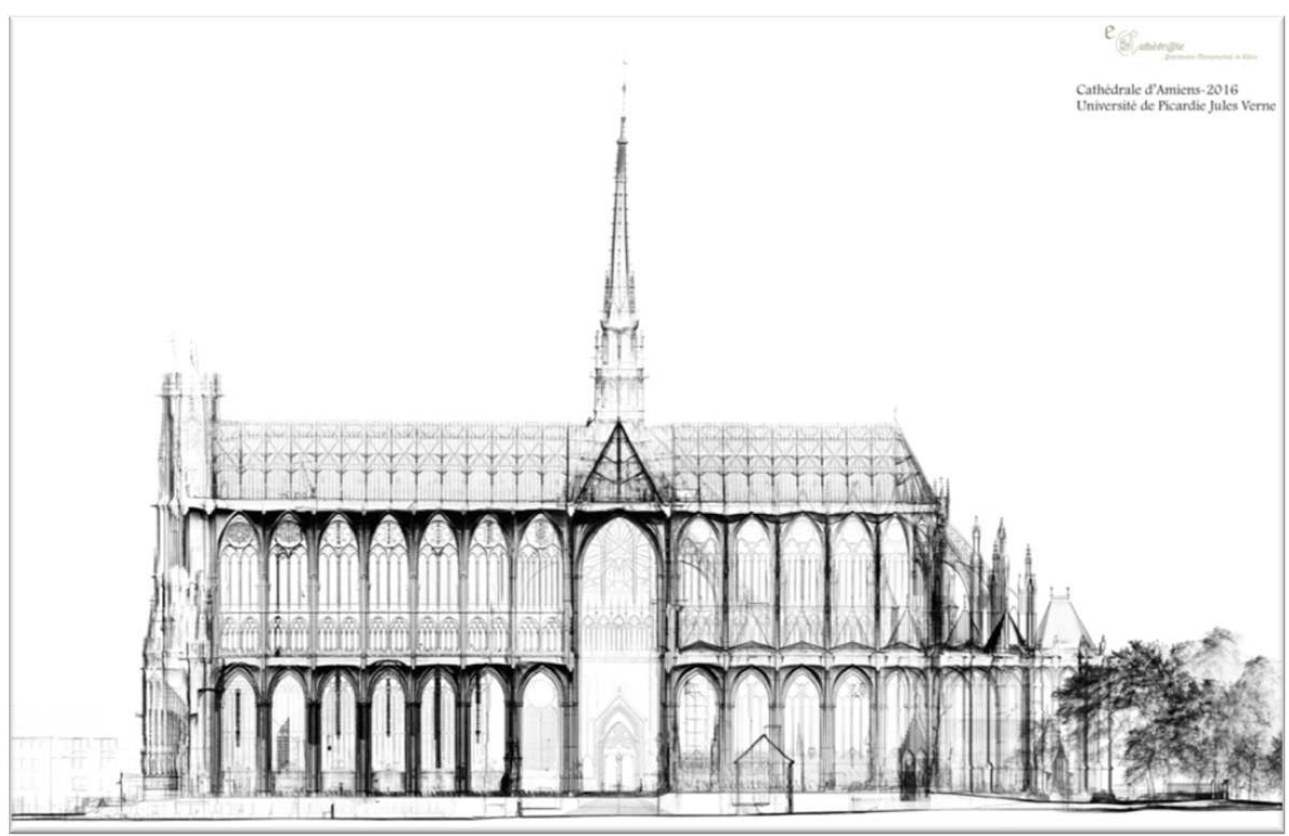

Plan d'élévation de la moitié nord (vue du sud). Cette vue superpose tout ce qui a été numérisé de l'intérieur et de l'extérieur en mode silhouette. Le rendu fait apparaître aussi bien les piliers, la voûte (intrados et extrados) que la charpente.

(c) Laboratoire MIS (programme E-Cathédr@le).

12 À partir du modèle réalisé, on peut également produire des extraits des parties cachées les moins accessibles du monument avec une précision et une résolution permettant la mesure comme on peut le constater sur la figure 4. Cet exemple est extrait d'une étude dans laquelle nous avons utilisé le modèle pour estimer les positions des impacts d'un obus datant de la Première Guerre mondiale 9 . Ces impacts se situent dans les combles (entre l'extrados et la couverture) de la deuxième travée sud du chœur. La figure 4.a donne une vue panoramique de ces combles. La figure 4.b montre une vue 3D de ce volume et la figure 4.c est un agrandissement de la zone de l'impact (fig. 4). 


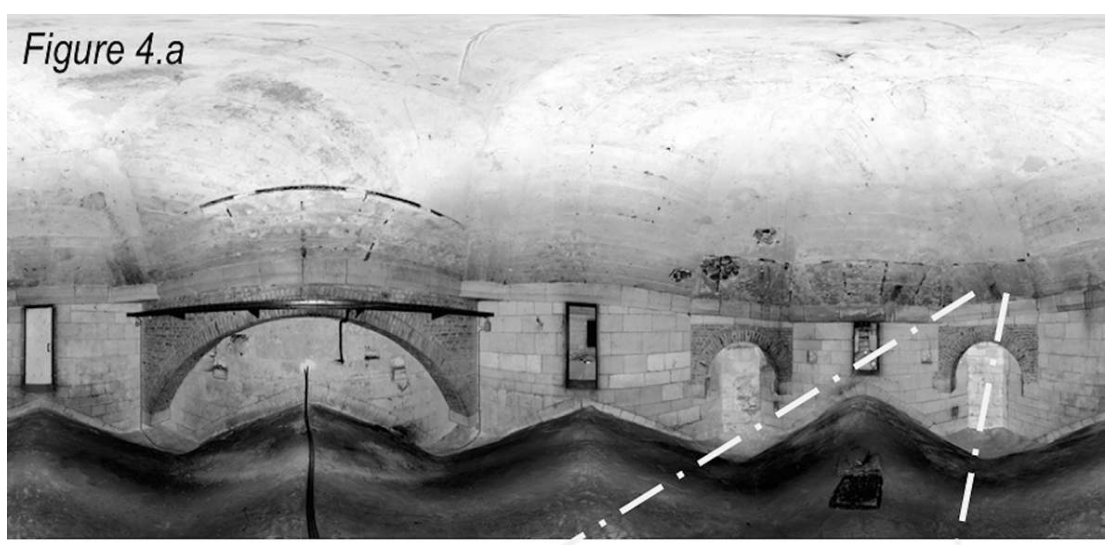

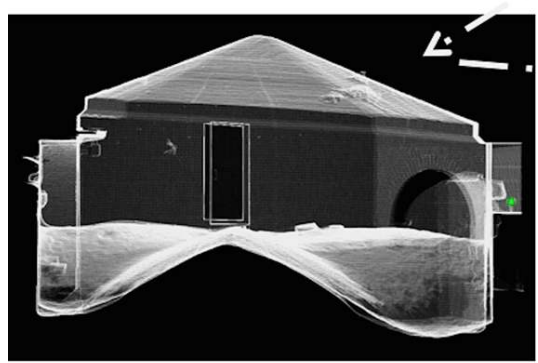

Figure 4.b

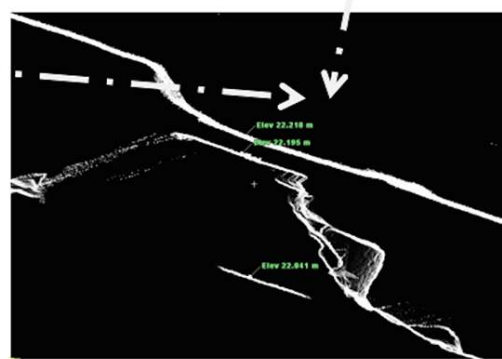

Figure 4.c

Détail d'un impact d'obus de la Première Guerre mondiale sur la couverture d'une chapelle avec les élévations (intérieur, couverture externe vue de l'intérieur et couverture extérieure).

(c) Laboratoire MIS (programme E-Cathédr@le).

Le dernier exemple montre l'intérêt d'un tel modèle pour observer finement la structure du monument. La figure 5.a est une vue de dessus de la moitié supérieure du modèle (la coupe a été faite juste au-dessus des voûtes des collatéraux pour améliorer la lisibilité de la figure). L'objectif est de montrer, et c'est une première, la géométrie des arcs-boutants. Du côté de la nef, la numérisation n'a pas encore été faite dans les hauteurs pour la partie nord, ce qui explique les manques. En revanche, pour le chœur, le modèle est complet et il nous donne la géométrie exacte de la structure externe. Les figures 5.b et 5.c sont des agrandissements qui mettent en évidence une irrégularité dans la disposition des arcsboutants. Il semble qu'elle ne soit pas due à des déformations mais à la disposition des piliers qui se situent en dessous. Cette irrégularité est présente de la même manière au nord du chœur (fig. 5). 

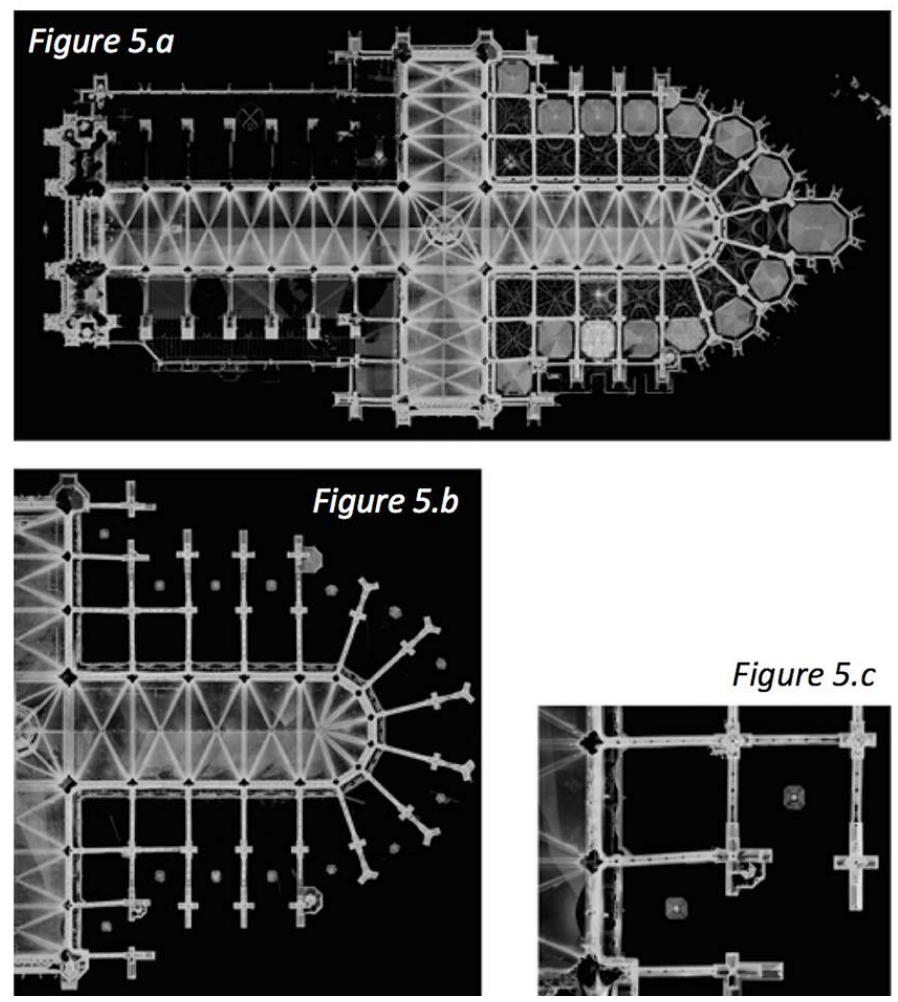

Coupe des arcs-boutants sud-est en vue de dessus et les parties supérieures des pinacles.

(c) Laboratoire MIS (programme E-Cathédr@le).

Dans l'état actuel du modèle, les surfaces réfléchissantes (vitraux par exemple), n'ont pas fait l'objet de technique de numérisation spécifique. Le Lidar C10 donne une information géométrique généralement cohérente grâce aux dépôts qui couvrent les vitraux, mais la texture devra être améliorée. Des pistes sont actuellement explorées, comme le recalage de photos sur la structure géométrique ${ }^{1011}$ ou bien encore la photogrammétrie. La figure 6 montre la validation de cette approche sur le portail sud de la cathédrale d'Amiens et sur une peinture murale avant et après recolorisation (fig. 6).

\section{Figure 6}
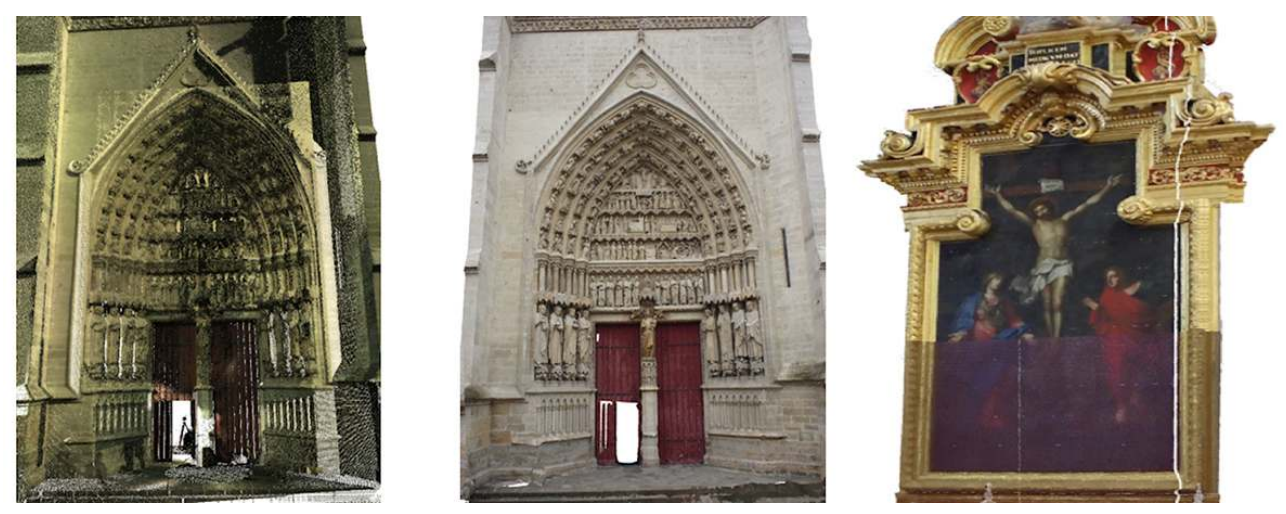

Recolorisation des points du modèle avec des photographies. Les deux images de gauche montrent l'état avant et après recolorisation. L'image de droite montre l'état du nuage issu du scanner (partie basse de l'image) et son état après recolorisation (partie haute).

(c) Laboratoire MIS (programme E-Cathédr@le). 
La photogrammétrie a été utilisée pour des zones pour lesquelles la lasergrammétrie présentait des occultations, et afin d'obtenir une résolution compatible avec les détails des sculptures. C'est le cas pour les façades, les portails et les statues. La figure 7 présente des résultats de la galerie des rois (façade occidentale). Pour assurer la proximité nécessaire à la prise des photographies, nous avons employé un dispositif de perche pour porter l'appareil photographique et les images ont été prises à partir de six niveaux. Cette campagne de numérisation ${ }^{12}$ s'est faite en collaboration avec l'IGN (Institut géographique national) et l'ENSG (École Nationale des Sciences Géomatiques) et c'est la suite logicielle Apero/MicMac ${ }^{13}$ qui a été utilisée pour les traitements. Tous les modèles réalisés par photogrammétrie sont géo-référencés dans un système unique de coordonnées (système RGF93). Les cartes de profondeur produites ont la même résolution que les images (entre $0,5 \mathrm{~mm}$ et $3 \mathrm{~mm}$ ) (fig. 7).

Figure 7
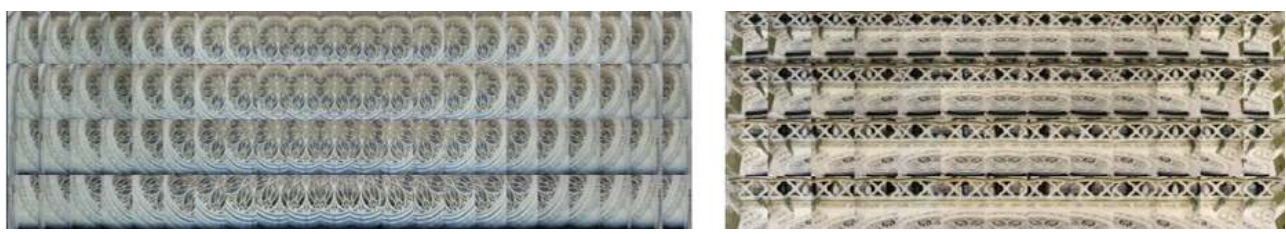

Images de la rose de la façade occidentale pour la photogrammétrie et extraits de la reconstruction.

(c) Laboratoire MIS (programme E-Cathédr@le).

Enfin, nous avons eu recours à une modélisation manuelle de la charpente de la flèche pour contourner la complexité de la structure à numériser d'une part et d'autre part pour disposer d'un modèle facilement exploitable d'un point de vue pédagogique. Ainsi, 1200 poutres ont été modélisées avec le logiciel Blender (fig. 8). Le reste de la charpente a été numérisé à l'aide d'un lidar (l'accès est facile et les occultations sont quasiment inexistantes parce que l'espace y est dégagé). 
Figure 8
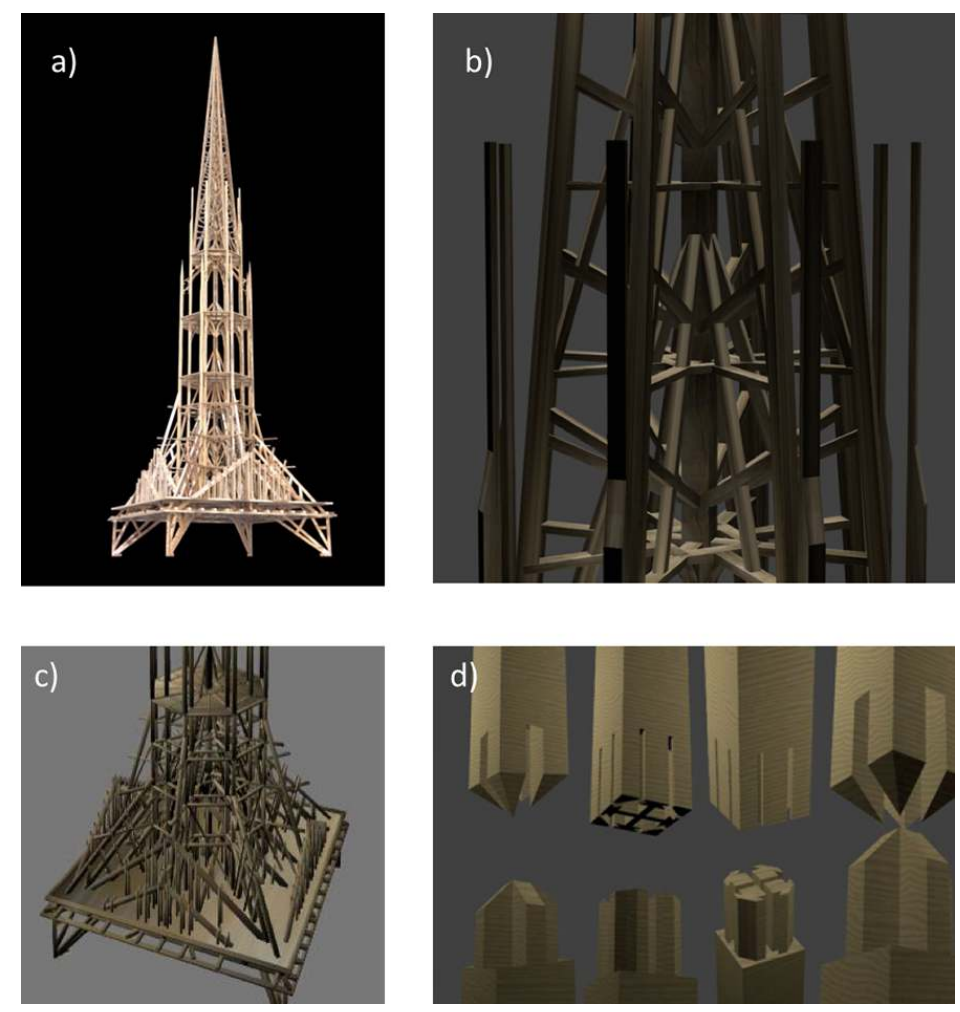

Rendu du modèle 3D de la flèche de la cathédrale d'Amiens. a) Vue globale de la charpente. b) Agrandissement de l'intérieur. c) Vue de la partie basse de la charpente. d) Détails des liaisons entre les poutres.

(c) Laboratoire MIS (programme E-Cathédr@le).

Dans la dynamique de E-Cathédr@le, nous avons mené d'autres projets de numérisation et de recherche sur la reconstruction 3D. La figure 9 donne un aperçu des monuments sur lesquels nous avons travaillé :

18 - la cathédrale de Beauvais. Ce projet a fait l'objet d'une collaboration avec le Vassar College et l'université Columbia (New York). Il a permis d'avoir un relevé texturé de l'ensemble de la cathédrale de Beauvais.

19 - la mosquée Hassan (Rabat, Maroc). Ces travaux se font en collaboration avec l'université de Rabat (Maroc). L'esplanade de la mosquée, qui date du XII siècle, a été numérisée en 2015. Nous avons ensuite proposé une reconstruction ${ }^{14}$ des parties manquantes basée sur les travaux de Jacques Caille ${ }^{15}$.

20 - l'église Saint Martin-aux-Bois (Oise). La numérisation a été effectuée en 2015 et poursuivie en 2016. Elle a concerné l'église et une partie des caves ${ }^{16}$.

21 - les «Belles du Nord », quatre statues de la Renaissance découvertes à Orchies lors de fouilles en 2015 (musée des Beaux-Arts, Lille).

22 Ces opérations, contrairement à la cathédrale d'Amiens, ont été plus limitées dans le temps (fig. 9). 


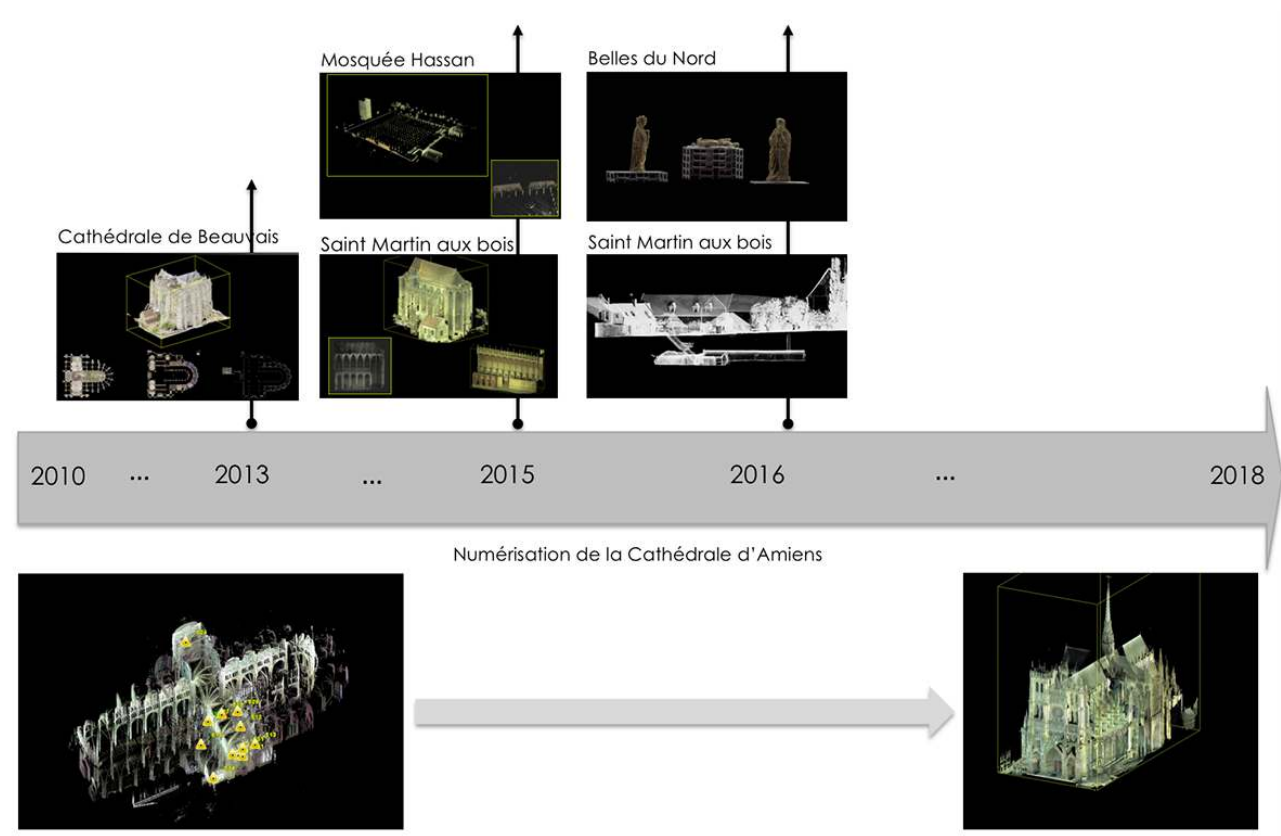

Autres monuments et sculptures numérisés dans le cadre du programme E-Cathédr@le.

(c) Laboratoire MIS (programme E-Cathédr@le).

\section{Retour d'expérience}

La numérisation d'un monument de la taille et de la complexité de la cathédrale d'Amiens pose des problèmes spécifiques de complexité et de nature différentes. Ces problèmes sont d'ordre technique, scientifique, administratif, juridique et financier. Parmi les problèmes techniques, on peut citer la question des données, leur mise à jour et la réalisation d'outils de navigation fluide dans les modèles. Sur le plan scientifique et pour ne citer que les aspects relevant des sciences du numérique, la quantification de la précision globale reste un problème complexe à cette échelle. Il en est de même de la question de la représentation des données (points, surfaces...) et du rendu (apparence, visualisation fluide, réalisme...). Les problèmes administratifs et juridiques rencontrés sont les autorisations d'accès au monument et au matériel à numériser ainsi que les questions de propriété et de droits sur les données. Dans le cas d'E-Cathédr@le, une convention impliquant l'université de Picardie-Jules Verne, la DRAC des Hauts-de-France et le Centre des monuments nationaux (CMN) a été établie. Elle permet au laboratoire MIS d'accéder au monument pour la numérisation et l'engage à fournir une copie des données au CMN. Une autre convention, élargie aux autres partenaires du programme (Amiens Métropole et la région Hauts-de-France) est en cours d'élaboration pour permettre l'exploitation des données. Enfin, sur le plan financier, il est évident que ce type de programme a un coût qui reste élevé. Dans le cas d'E-Cathédr@le, les financements proviennent essentiellement de l'université de Picardie-Jules Verne, de la région Hautsde-France, du fonds FEDER, de l'ANR, d'Amiens Métropole et de fonds privés (mécénat).

Au-delà de ces difficultés et d'E-Cathédr@le, nous souhaiterions à nouveau insister sur la nécessité d'inscrire ce genre de programme dans une temporalité compatible avec les 
exigences et le cahier des charges établis à priori, car ce qui est important et urgent, ce n'est pas tant la preuve du concept, mais la numérisation systématique et complète ellemême ainsi que la valorisation du modèle. Il nous semble essentiel de créer des collaborations entre chercheurs en sciences du numérique, historiens de l'art, historiens, acteurs $\mathrm{du}$ patrimoine (conservateurs, architectes du bâtiment ...), décideurs et collectivités pour que les différents ingrédients nécessaires à une véritable réussite soient pris en compte. Cette réussite se mesure aussi bien par la numérisation elle-même que par la valorisation et la pérennisation des données. Sur ce dernier point, il est nécessaire qu'une prise de conscience se fasse pour que les professionnels de l'archivage se saisissent de la question de la conservation des données 3D. Comme pour les supports papier, toutes les données numériques ne sont pas à conserver, mais certains projets de numérisation ont abouti à la réalisation de corpus exceptionnels qu'il est urgent de traiter de la même manière que tout autre document scientifique.

Enfin, nous constatons encore une insuffisance de la reconnaissance des travaux relevant de ce domaine. D'une part, parce que ces travaux sont nécessairement pluridisciplinaires et que la pluridisciplinarité, pourtant louée et appelée partout, n'en demeure pas moins périlleuse dans un système d'évaluation de la recherche très sectorisé. Ce point constitue un frein à la prise en charge de la numérisation 3D massive et à la constitution de corpus aboutis par les acteurs académiques.

\section{Conclusion}

Lors du démarrage du programme E-Cathédr@le en 2010, nous avions clairement annoncé que la numérisation de la cathédrale d'Amiens et le développement de travaux de recherche pluridisciplinaires allaient être notre priorité. Cette ligne directrice est une garantie pour que le modèle réalisé réponde aux exigences fixées (résolution, précision, complétude) et pour qu'il ne soit pas réduit à un «corpus des placards ». Le relevé 3D dont nous disposons actuellement représente environ $80 \%$ du monument. Les parties manquantes sont les plus difficiles d'accès (cavités, combles, ...). Nous menons actuellement des travaux de recherche qui ont pour objectif de proposer des solutions pour rendre plus fiable et plus aisée la numérisation de ces parties complexes ainsi que des systèmes robotisés plus adaptés à la numérisation massive.

Nous avions également prévu des restitutions de l'état d'avancement du programme et la mise en place de dispositifs d'accès aux résultats obtenus. Les restitutions se font sous forme de conférences et de démonstrations avec l'accès au modèle 3D grâce à des dispositifs de visualisation 2D et 3D et d'immersion. Pour cela, nous avons développé des systèmes adaptés au rendu directement à partir des nuages de points, de manière à éviter le recours au maillage qui altère les données. La réalisation de ces dispositifs est centrale car elle représente un point de passage obligé pour que les corpus constitués soient exploitables et utilisables aussi bien par les chercheurs, les étudiants que le grand public.

Les exemples de travaux et de perspectives que nous avons mentionnés existent, à des échelles différentes, dans de nombreux laboratoires et organismes. Cela démontre l'intérêt porté à la question du recours au numérique pour le patrimoine monumental. Cet intérêt doit viser la production de modèles 3D. Les outils actuellement disponibles permettent d'envisager une numérisation systématique du patrimoine, même si ces outils ont vocation à être améliorés et complétés. Il reste à encourager, soutenir, inciter et coordonner les initiatives. Ceci pourrait se faire par la création d'une structure nationale 
pérenne pour assurer cette mission. Nous l'appelons de tous nos vœux pour qu'enfin, le patrimoine architectural soit entièrement in silico.

\section{NOTES}

1. - 'In silico' is a neologism coined to denote the heritage that exists inside the computer.

2. - Voir le site : http://www.gamsau.map.archi.fr/3D-monuments/ [consulté le 11/04/2019].

3. - Voir sur le site: www.culture.gouv.fr/content/download/182631/1996185/version/2/.../ GT_4-2.pdf [consulté le 11/04/2019].

4. - ABERGEL, Violette, BENISTANT, Pascal, BERGEROT, Laurent, BERNARD, Jean-François, BOHBOT, Hervé, et al. Livre blanc du Consortium 3D SHS. France, 2017.

5. - Voir le site : https://mis.u-picardie.fr/e-cathedrale/ [consulté le 11/04/2019].

6. - MORBIDI, Fabio, MOUADDIB, El Mustapha. «E-Cathédr@le : numérisation de la cathédrale d'Amiens et reconstruction des trajectoires balistiques de l'artillerie allemande en avril 1918 ». Actes du colloque « Cathédrales en guerre, $\mathrm{XVI}^{\mathrm{e}}-\mathrm{XX}^{\mathrm{e}}$ siècles ».

7. - Voir le site: https://home.mis.u-picardie.fr/ mouaddib/JS/ [consulté le 11/04/2019]. HABIBI, Zaynab, CARON, Guillaume, MOUADDIB, El Mustapha. « Assistive visual framing ». Digital Heritage 2015, Grenade, 28 sept. - 2 oct. 2015. HABIBI, Zaynab, MOUADDIB, El Mustapha, CARON, Guillaume. "Good feature for framing ». IEEE/RSJ International Conference on Intelligent Robots and Systems, Hambourg, 28 sept. - 2 oct. 2015. LECLET-GROUX, Dominique, CARON, Guillaume. " Toward the adaptive and context-aware Serious Game design ». IEEE International Conference on Advanced Learning Technologies, ICALT, Athènes, juillet 2014. LECLET-GROUX, Dominique, CARON, Guillaume, MOUADDIB, El Mustapha, ANGHOUR, A. " A Serious Game for 3D Cultural Heritage ». IEEE Digital Heritage International Congress, DH, p.409-412, Marseille, octobre 2013. CARON, Guillaume, LECLET-GROUX, Dominique, CROMBEZ, Nathan, MOUADDIB, El Mustapha. «From Heritage Building Digitization To Computerized Education ». 6th International Congress on Science and Technology for the Safeguard of Cultural Heritage in the Mediterranean Basin. Athènes, octobre 2013.

8. - BALTSAVIAS, Emmanuel P. "A comparison between photogrammetry and laser scanning ". ISPRS Journal of Photogrammetry and Remote Sensing, vol. 54/2, p. 83-94.

9. - MORBIDI, Fabio, MOUADDIB, El Mustapha. «E-Cathédr@le : numérisation de la cathédrale d'Amiens et reconstruction des trajectoires balistiques de l'artillerie allemande en avril 1918 ». Actes du colloque «Cathédrales en guerre, $\mathrm{XVI}^{\mathrm{e}}-\mathrm{XX}^{\mathrm{e}}$ siècles ». Op. Cit.

10. - CROMBEZ, Nathan, CARON, Guillaume, MOUADDIB, El Mustapha. «3D point cloud model colorization by dense registration of digital images ». 3D Virtual Reconstruction and Visualization of Complex Architectures, 3DARCH, ISPRS workshop, fév. 2015, Avila.

11. - CROMBEZ, Nathan, CARON, Guillaume, MOUADDIB, El Mustapha. "Colorisation photoréaliste de nuages de points $3 \mathrm{D}$ ». Orasis, Congrès des jeunes chercheurs en vision par ordinateur, juin 2013, Cluny, France.

12. - HÉNO, Raphaël, CHANDELIER, Laure, SCHELSTRAETE, D. «Costless platform for high resolution stereoscopic images of a high gothic façade». International Archives of the Photogrammetry, Remote Sensing and Spatial Information Sciences, vol. XXXIX-B5, 2012 XXIIth ISPRS Congress, 25 août - 1er sept. 2012, Melbourne. 
13. - PIERROT-DESEILLIGNY, Marc, CLERY, Isabelle. «APERO, an Open Source Bundle Adjusment Software for Automatic Calibration and Orientation of a Set of Images ». Proceedings of the ISPRS Commission V Symposium, Image Engineering and Vision Metrology, Trente, 2-4 mars 2011.

14. - ACHAKIR, Farouk, DESEILlignY, Marc, EL FKIHI, Sanaa, EL MGHARI, Min, ETTARID, Mohamed, MOUADDIB, El Mustapha, RADGUI, Amina. "The Hassan mosque at the digital era ». Frontiers in Science and Engineering - International Journal. Hassan II Academy of Science and Technology, 2017.

15. - CAILLE, Jacques. «La Mosquée de Hassan à Rabat ». Editions Arts et Métiers Graphiques pour l'Institut des Hautes Etudes Marocaines, 1954.

16. - DOPERE, Franz et MOUADDIB, El Mustapha. L'abbatiale de Saint-Martin-aux-Bois. Archéologie du bâti et numérisation 3D, nouvelles perspectives de recherche. Actes des journées d'étude des mois de mai 2015 et 2016, vol. 31, 2018.

\section{RÉSUMÉS}

De nombreux projets en rapport avec la numérisation et la modélisation 3D de monuments historiques ont vu le jour ces dernières années, bénéficiant de l'accélération et de la fiabilisation des moyens techniques de relevé 3D et de traitement des données. La démocratisation de ces techniques et les avancées méthodologiques (notamment en photogrammétrie/vision par ordinateur) ont également participé à cet engouement. Construire une empreinte digitale fidèle et complète des monuments puis l'augmenter avec des connaissances textuelles pour en faire un objet d'étude et de recherche pluridisciplinaire, telle a été notre motivation principale pour concevoir et mettre en œuvre le programme E-Cathédr@le avec la cathédrale d'Amiens comme site privilégié. Nous nous sommes fixés comme objectif d'en faire un relevé complet (intérieur et extérieur), fidèle, précis, résolu et photométriquement réaliste. Chacun de ces critères recouvre une signification scientifique très précise et qui a des conséquences importantes sur le mode opératoire, sur la quantité des données à enregistrer et à manipuler. Ces critères nous ont également parfois obligés à mettre au point des modalités d'acquisition nouvelles et adaptées. Lancé en 2010, pour une durée estimée à quinze ans, ce programme a déjà permis la numérisation de plusieurs monuments en France (dont la cathédrale d'Amiens) et au Maroc grâce à un projet bilatéral de recherche. Ces modèles numériques font l'objet d'études et de travaux de recherche pluridisciplinaires, impliquant notamment l'informatique, la robotique, l'histoire de l'art et l'archéologie. Nous nous proposons ici de décrire ce programme, d'exposer les résultats de la numérisation de la cathédrale d'Amiens qui révèlent de manière inhabituelle des plans et des coupes d'élévations extrêmement précis et permettant de mieux " lire » l'architecture de ce monument. Nous livrerons également un retour d'expérience à partir des huit premières années du programme et évoquerons les difficultés posées par les projets de numérisation du patrimoine monumental.

Several digital heritage projects for the creation of 3D models of historic buildings have emerged during recent years. Such projects have been stimulated by some significant advances in digital surveying technologies and in data processing. The wider diffusion of these techniques and several other methodological advances, notably in computer vision and photogrammetry, have also contributed to this phenomenon. The goal of the E-Cathédr@le programme, developed by our research group and centred on Amiens cathedral in France, is to keep a record of a historic 
monument which is as accurate and reliable as possible, and to enrich this record with textual information providing the basis for multidisciplinary research. While the objective is to cover the totality of the monument, both inside and out, and to take high-resolution and photometricallyrealistic measurements, each of these criteria has its own scientific pertinence and an important impact on the modus operandi and on the quantities of data to be recorded and managed. These challenges forced us to design new adapted modalities of data capture. The programme was launched in 2010 and is expected to last 15 years. It has already allowed for the digitization of several other monuments in France and in Morocco, thanks to a bilateral research project. These digital models have been shared and widely used for multidisciplinary research in computer science, robotics, art history and archaeology. In this paper, we describe the E-Cathédr@le programme and we present its main results. In particular, we show how our 3D model of the monument allows for the easy creation of accurate plans and cross sections of the architecture. This in turn allows for the body of the building to be visualised in an innovative way. We conclude the paper with a discussion of the lessons learned during the first eight years of the programme, and of the main challenges and opportunities involved in digital heritage projects.

\section{INDEX}

Mots-clés : numérisation, patrimoine architectural, modélisation 3D, cathédrale d'Amiens

Keywords : digitalisation, architectural heritage, 3D models, cathedral of Amiens

\section{AUTEURS}

\section{EL MUSTAPHA MOUADDIB}

Professeur des universités, Université de Picardie Jules Verne mouaddib@u-picardie.fr

\section{GUILLAUME CARON}

Maître de conférences, Université de Picardie Jules Verne guillaume.caron@u-picardie.fr

\section{DOMINIQUE GROUX-LECLET}

Maître de conférences, Université de Picardie Jules Verne dominique.groux@u-picardie.fr

\section{FABIO MORBIDI}

Maître de conférences, Université de Picardie Jules Verne fabio.morbidi@u-picardie.fr 